\title{
VIBRATIONAL AND ROTATIONAL COOLING OF ELECTRONS BY WATER VAPOR
}

\author{
T. E. CRAVENS \\ Space Physics Research Laboratory, University of Michigan, Ann Arbor, MI 48109, U.S.A. \\ and
}

A. KOROSMEZEY

Central Research Institute for Physics, Budapest, Hungary

(Received in final form 11 March 1986)

\begin{abstract}
The cooling of electrons by vibrational and rotational excitation of water molecules plays an important role in the thermal balance of electrons in cometary ionospheres. The energy loss function for rotational excitation and de-excitation of $\mathrm{H}_{2} \mathrm{O}$ by electron impact is calculated theoretically. The rotational cooling rate is calculated using this loss function for a wide range of electron and neutral temperatures. The vibrational cooling rate is calculated using measured values of electron impact vibrational excitation cross sections. Analytical formulae are provided for some of the cooling rates. The interaction of ions with $\mathrm{H}_{2} \mathrm{O}$ molecules is also discussed and a formula is suggested for the momentum transfer collision frequency.
\end{abstract}

\section{INTRODUCTION}

Cometary atmospheres are very extensive due to the virtual absence of gravity and the major neutral constituent is thought to be water (Delsemme, 1982). The solar wind interacts very strongly with cometary atmospheres and/or ionospheres (Ip and Axford, 1982; Schmidt and Wegmann, 1982; Mendis et al., 1985; Galeev et al., 1985). The electron temperature either in the solar wind or the ionosphere depends on both heating and cooling processes, as well as heat transport by conductivity. The electron heat balance is an important part of the overall solar wind interaction with comets. The dynamics of the ionosphere also depend on the electron temperature structure (Marconi and Mendis, 1984; Cravens et al., 1983). Wallis and Ong (1976) discussed many of the physical processes relevant to the energy balance of electrons near comets. Ashihara (1976) calculated photoelectron fluxes in a cometary ionosphere, and photoelectrons are a major contributor to the heating of the ionospheric electrons. Marconi and Mendis (1984) and Korosmezey (1985) calculated electron temperatures in the cometary ionosphere using the electron-water rotational and vibrational cooling rates of Ashihara (1975).

Cooling rates are a necessary part of any electron temperature calculation and for comets the cooling is especially efficient because the electron impact cross sections for rotational and vibrational excitation are very large for polar molecules like $\mathrm{H}_{2} \mathrm{O}$. In this paper, we will present the results of new cooling rate cal- culations which are more accurate than those of Ashihara (1975). We will employ measured vibrational excitation cross sections (i.e. Seng and Linder, 1976; Rohr, 1977). We will use the results of an improved energy loss calculation in a rotational cooling rate calculation. This energy loss function (or stopping power) should also be useful for calculations of photoelectron and solar wind electron fluxes at low energies.

\section{ENERGY LOSS FUNCTION FOR ROTATIONAL EXCITATION AND DE-EXCITATION BY ELECTRON IMPACT}

Very little experimental data is available for the rotational excitation of $\mathrm{H}_{2} \mathrm{O}$ by electrons (Jung et al., 1982). However, theoretical calculations have shown that the cross sections for rotational excitation are very large for molecules with large permanent dipole moments such as $\mathrm{H}_{2} \mathrm{O}$ (cf. Takayanagi and Itikawa, 1970; Itikawa, 1972; Jain and Thompson, 1983). Itikawa (1972) derived cross section formulae for electron impact rotational transitions in asymmetric-top molecules (including $\mathrm{H}_{2} \mathrm{O}$ ) using the first Born approximation. The more elaborate theoretical calculations of Jain and Thompson (1983) were only carried out for a few of all the possible rotational transitions required for an energy loss function calculation; however, they demonstrated that the Born approximation is very good for $\mathrm{H}_{2} \mathrm{O}$ and that quadrupole transitions are much less important than dipole transitions. We will use the Itikawa cross section formula for dipole transitions. 
The energy levels of an asymmetric-top molecule can be labeled with the rotational quantum number, $J$, and a pseudo quantum number $\tau$, which can take on values from $-J$ up to $J$. The rotational eigenfunctions of an asymmetric rotor belong to the fourgroup $V(\xi, \eta, \zeta)\left(C_{2 v}\right.$ symmetry) with the possible symmetry species of $\mathbf{A}, \mathbf{B}_{\xi}, \mathbf{B}_{\eta}, \mathbf{B}_{\zeta}$ (cf. Itikawa, 1972; Jain and Thompson, 1983). $\zeta, \xi$ and $\eta$ are the coordinate axes corresponding to the three principle axes of the ellipsoid of inertia of the molecule. For dipole transitions, the allowed transitions are:

$$
\begin{gathered}
\Delta J=0, \pm 1 \quad\left(\text { but } \mathbf{J}=0 \leftarrow / \rightarrow J^{\prime}=0\right) \\
\mathrm{A} \leftrightarrow B_{\zeta} \cdot(\text { even } \tau \text { levels }) \\
\mathbf{B}_{\xi} \leftrightarrow \mathbf{B}_{\eta} \quad \text { (odd } \tau \text { levels) }
\end{gathered}
$$

Allowed dipole transitions are not permitted between states with even values of $\tau$ and states with odd values of $\tau$.

The energies of the $J, \tau$ levels (i.e. $E_{J, \tau}$ ) were taken from Dalby and Nielson (1956) who derived them

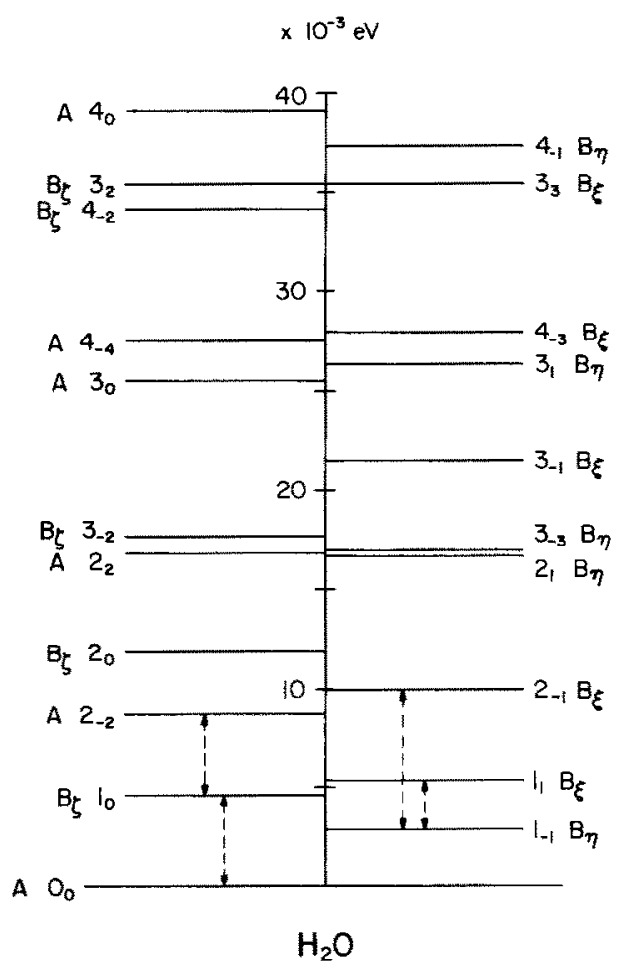

Fig. 1. ENERGY LEVEL DIAGRAM FOR THE ROTATIONAL STATES OF WATER.

Each level is labeled with $J$ and $\tau$ and with its symmetry species. Four of the many possible transitions are indicated by the dashed lines. using infra-red data. A rotational level diagram for the lower $J$ levels of $\mathrm{H}_{2} \mathrm{O}$ is shown in Fig. 1. The even levels have a statistical weight of $1 / 4$ and the odd levels $3 / 4$. The statistical weight of a particular $J, \tau$ level also includes the factor $(2 J+1)$. The relative population of a level for a rotational temperature, $T$, is

$$
\begin{aligned}
f(J, \tau, T)=(1 / Q(T)) & \left(2-(-1)^{|\tau|}\right) \\
& \times(2 J+1) \exp \left\{-E_{j, \tau} / k T\right\}
\end{aligned}
$$

where the partition function is

$$
Q(T)=\sum_{J=0}^{J}(2 J+1) \sum_{\tau=-J}^{\tau=J}\left(2-(-1)^{|\tau|}\right)
$$

$$
\exp \left\{-E_{J_{x} / k} / k T\right\}
$$

We calculated a value of 171 for the partition function at $T=293 \mathrm{~K}$, whereas van Vleck (1947) obtained a value of 170 .

The dipole cross section formula from Itikawa (1972) is

$$
\begin{aligned}
\sigma\left(J, \tau \rightarrow J^{\prime}, \tau^{\prime}\right)= & \left(8 \pi / 3 k^{2}\right)\left(2 J^{\prime}+1\right) D^{2} \\
& \times\left\langle J^{\prime} \tau^{\prime} \mid J \tau\right\rangle^{2} \ln \left|\left(k+k^{\prime}\right) /\left(k-k^{\prime}\right)\right|
\end{aligned}
$$

$J$ and $\tau$ are the quantum numbers for the initial state of the transition and $J^{\prime}$ and $\tau^{\prime}$ are for the final state. $k$ and $k^{\prime}$ are the initial and final wave numbers for the electron. $D$ is the dipole moment of the water molecule ( $D=0.728$ atomic units). $\left\langle J^{\prime} \tau^{\prime} \mid J \tau\right\rangle$ is the line strength of the transition and is closely related to the optical line strength, $S$, for which we adopted values from Cross et al. (1944) and King et al. (1947):

$$
S=\left(2 J^{\prime}+1\right)(2 J+1)\left\langleJ ^ { \prime } \left\{\left|J_{\tau}\right\rangle^{2} .\right.\right.
$$

The rotational cross sections calculated using equations (3) and (4) are very large at low energies (e.g. typically $1-3 \times 10^{-13} \mathrm{~cm}^{2}$ for energies of about $10^{-2}$ eV). Figure 2 shows cross sections for a few transitions (from Fig. 4 of Itikawa, 1972).

Since a very large number of $J, \tau$ levels can be excited or de-excited even by very low energy electrons for moderately high rotational temperatures, the interaction of electrons with $\mathrm{H}_{2} \mathrm{O}$ is most efficiently described using a loss function in a manner analogous to that presented in Henry and McElroy (1969) for diatomic molecules. The loss function, $L(E, T)$, for an electron with an energy $E$ in water vapor with temperature $T$ is :

$$
L(E, T)=\sum_{B} \sum_{J} \sum_{\mathrm{\tau}} \sum_{\mathrm{\tau}^{\prime}}\left[E_{J^{\prime}(J, B), \tau^{\prime}}-E_{J, \tau}\right] \times\{\}
$$




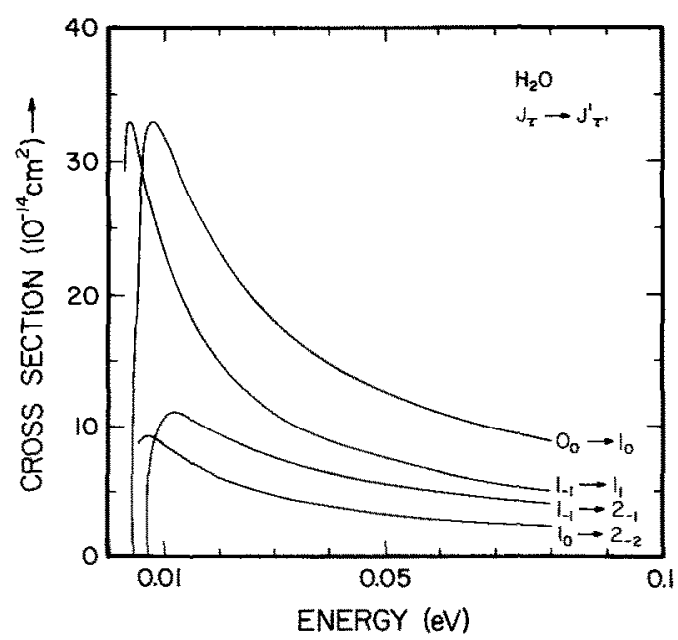

FIG. 2. ELECTRON IMPACT CROSS SECTIONS FOR SELECTED TRANSITIONS IN $\mathrm{H}_{2} \mathrm{O}$ AS A FUNCTION OF ELECTRON ENERGY. Adapted from Fig. 4 of Itikawa (1972).

where the term in brackets is:

$$
\begin{aligned}
\{\}=f(J, \tau ; T) \sigma\left(J, \tau \rightarrow J^{\prime}(J, B), \tau^{\prime} ; E\right) & \\
& -f\left(J^{\prime}(J, B), \tau^{\prime} ; T\right) \sigma\left(J^{\prime}(J, B), \tau^{\prime} \rightarrow J, \tau ; E\right) .
\end{aligned}
$$

$B$ is an index indicating the branch or type of transition. With the selection rules from equation (1), six types of transitions are possible giving $\mathrm{Q}, \mathrm{R}$ and $\mathrm{P}$ branches for both even and odd levels. The cross sections are non-zero only for those combinations of $B, J, \tau$ and $\tau^{\prime}$ that obey these selection rules. However, $\mathrm{R}$ and $\mathbf{P}$ transitions are taken care of together, hence $B$ ranges from 1 through $4 . J^{\prime}$ is determined by the values of $J$ and $B$. $\tau$ and $\tau^{\prime}$ vary from $-J$ to $+J$ and from $-J^{\prime}$ to $+J^{\prime}$, respectively. The cross sections are taken from the Born formula of Itikawa (1972) (equations 3 and 4), and the relative density for a given level is given by equation (2).

Calculated loss functions for several values of the neutral temperature are shown in Fig. 3. The maximum value of $J$ used in equation (5), $J_{\max }$, was chosen to be 10 for all these calculations. This value of $J_{\max }$ limits the accuracy of calculations for temperatures greater than about $500 \mathrm{~K}$. The estimated convergence error for this $J_{\max }$ is less than $0.04 \%$ for $100 \mathrm{~K}$, about $1.4 \%$ for $300 \mathrm{~K}$, and about $6.5 \%$ for $500 \mathrm{~K}$.

Consider the loss function for $T=500 \mathrm{~K} . L(E$, $500 \mathrm{~K}$ ) has a maximum near an energy of $0.07 \mathrm{eV}$, goes through zero at $0.04 \mathrm{eV}$, and is negative for energies less than $0.04 \mathrm{eV}$. A negative loss function means that an incident electron gains energy on the average, which can happen if there are more de-excitation than excitation collisions. The "zero" of the function $L(E, T)$ depends strongly on $T$, increasing as $T$ increases. The peak value of $L(E, T)$ increases as $T$ decreases. The loss function curves are rather smooth for larger values of $T$ because a large number of both excitation and de-excitation collisions contribute to the sums in equation (5). However, for very low temperatures such as $T=10 \mathrm{~K}$, only the lowest couple of levels are populated and only a few transitions are important, and the loss functions show more structure due to the energy dependence of individual cross sections. At $10 \mathrm{~K}$, the fractional population of the $0_{0}\left(J\right.$ and $\tau$, respectively), $1_{-1}, 1_{0}$ and $l_{1}$ levels are about $0.7,0.2,0.01$ and 0.01 , respectively. Only the $0_{0} \leftrightarrow 1_{0}$ and the $1_{\ldots .} \leftrightarrow 1_{1}$ transitions make a significant contribution to equation (5). For $E$ less than the threshold for the former transition $(E<0.0023 \mathrm{eV})$, only de-excitation collisions are possible and $L(E, 10 \mathrm{~K})$ is negative. For $E$ greater than the threshold of the second transition $(E>0.0046 \mathrm{eV})$ excitation becomes possible for both transitions.

The rotational loss functions are almost independent of $T$ for incident electron energies greater than about $0.5 \mathrm{eV}$. The loss function for $E>0.5 \mathrm{eV}$ and for $T=300 \mathrm{~K}$ can be approximated by:

$$
L(E, 300 \mathrm{~K})=5.20 \times 10^{-17} / E^{0.9} .
$$

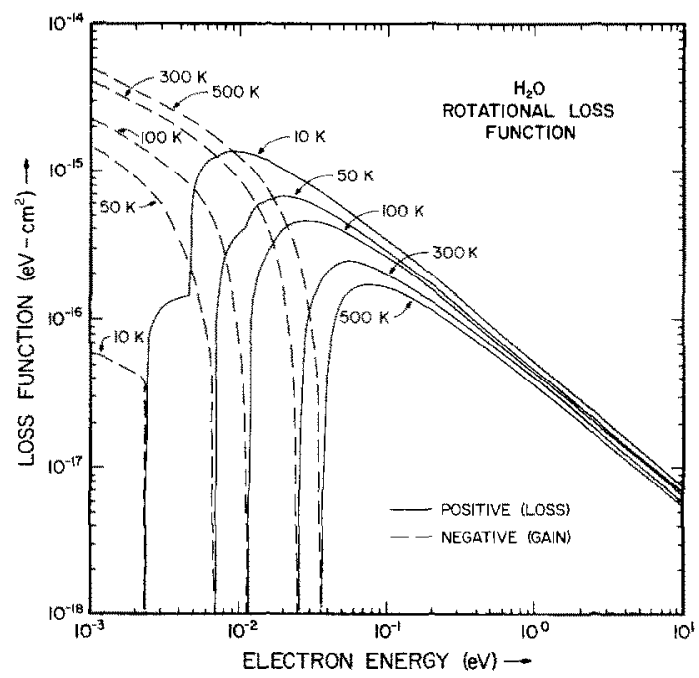

Fig. 3. CALCULATED ROTATIONAL LOSS FUNCTIONS (OR STOPPING POWER) FOR ELECTRONS IN WATER VAPOR VERSUS ELECTRON ENERGY.

Each loss function curve is labeled with the rotational temperature used. The solid lines represent energy lost by the electrons and the dashed lines represent energy gained. 
However, vibrational rather than rotational excitation of $\mathrm{H}_{2} \mathrm{O}$ is a more important energy loss process at higher energies.

\section{ROTATIONAL COOLING OF ELECTRONS BY WATER YAPOR}

The electron cooling rate for a process can be obtained by integrating the energy loss function for that process with the flux of thermal electrons as a function of energy assuming that the distribution is a Maxwellian with an electron temperature $T_{\mathrm{e}}$. The rotational cooling rate for $\mathrm{H}_{2} \mathrm{O}, R_{\mathrm{r}}\left(T_{\mathrm{e}}, T_{\mathrm{n}}\right)$, as a function of electron temperature and neutral temperature, $T_{\mathrm{n}}$, is :

$R_{\mathrm{r}}\left(T_{\mathrm{e}}, T_{\mathrm{n}}\right)=\int_{0}^{\infty} L\left(E, T_{\mathrm{n}}\right) v f_{\mathrm{e}}\left(E, T_{\mathrm{e}}\right) \mathrm{d} E\left(\mathrm{eV} \mathrm{cm}^{3} \mathrm{~s}^{-1}\right)$

where $L\left(E, T_{\mathrm{n}}\right)$ is the rotational loss function (described in section 2), $f_{\mathrm{e}}\left(E, T_{\mathrm{e}}\right)$ is a Maxwellian electron distribution function normalized to unity, and $v=\left(2 E / m_{\varepsilon}\right)^{1 / 2}$ is the electron velocity as a function of encrgy. $R_{\mathrm{r}}\left(T_{\mathrm{e}}, T_{\mathrm{n}}\right)$ multiplied by both the density of thermal electrons and the $\mathrm{H}_{2} \mathrm{O}$ density gives the rotational cooling rate per unit volume (units of $\mathrm{eV} \mathrm{cm}^{-3} \mathrm{~s}^{-1}$ ).
Cooling rates were calculated by numerically evaluating the integral in equation (7) and using the calculated loss functions from section 2. Cooling rate curves are shown as functions of $T_{\mathrm{e}}$ in Fig. 4 for several values of the neutral temperature. The cooling rates are almost constant for the higher values of $T_{\mathrm{e}}$ shown in Fig. 4, decreasing only very slightly with increasing $T_{\mathrm{e}}$ due to the decrease in the cross sections at higher energies. The cooling rates are expected to be zero for $T_{\mathrm{e}}$ equal to $T_{\mathrm{n}}$; actually, the zeros of the calculated curves are very slightly off from this due to numerical error. The cooling rates are negative for values of $T_{\mathrm{e}}<T_{\mathrm{n}}$; that is, the thermal electrons are heated by their interaction with water vapor.

These cooling rates are extremely large because the $\mathrm{H}_{2} \mathrm{O}$ molecule has a large permanent dipole moment. For comparison, the rotational cooling rate at $T_{\mathrm{e}}=1000 \mathrm{~K}$ and $T_{\mathrm{n}}=300 \mathrm{~K}$ for the non-polar molecule, $\mathrm{H}_{2}$, is close to $8 \times 10^{-12} \mathrm{eV} \mathrm{cm}^{3} \mathrm{~s}^{-1}$ (Waite and Cravens, 1981), whereas the $\mathrm{H}_{2} \mathrm{O}$ cooling rate from Fig. 4 is about 100 times larger.

Cooling rates were calculated for approximately twice as many neutral temperature values as those shown in the figure. These calculated rates were fit with the following analytical function of $T_{\mathrm{e}}$ and $T_{11}$ :

$$
R_{\mathrm{r}}\left(T_{\mathrm{e}}, T_{\mathrm{n}}\right)=\left\{a+b \ln \left(T_{\mathrm{c}} / T_{\mathrm{n}}\right)\right\}\left\{\left(T_{\mathrm{c}}-T_{\mathrm{n}}\right) / T_{\mathrm{e}}^{\mathrm{S} / 4}\right\}
$$

where the coefficients are the following functions of

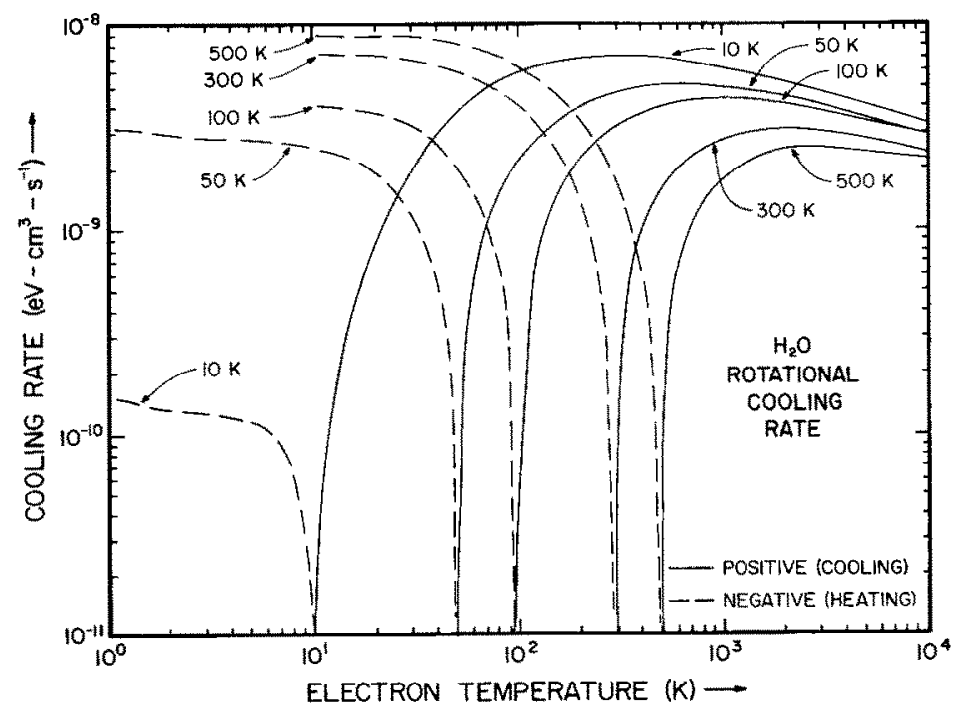

Fig. 4. Calculated rotational cooling rates for Maxwellian electrons in water vapor versus ELECTRON TEMPERATURE.

Each curve is labeled with the rotational temperature used. The solid lines represent cooling of the electrons and the dashed lines heating of the electrons. 
$T_{n}:$

$$
\begin{aligned}
& a=1.052 \times 10^{-8}+6.043 \times 10^{-10} \ln T_{\mathrm{n}} . \\
& b=4.18 \times 10^{-9}+2.026 \times 10^{-10} \ln T_{\mathrm{n}} .
\end{aligned}
$$

Notice that this cooling rate function is automatically zero at $T_{\mathrm{e}}-T_{\mathrm{n}}=0$. Equation (8) is accurate to within $12 \%$ for $120 \mathrm{~K}<T_{\mathrm{e}}<4000 \mathrm{~K}$ and for $T_{\mathrm{n}}>30 \mathrm{~K}$, although less accurate cooling rates can be obtained outside this range. For example, the error due to the fit is less than a factor of 2 for $T_{\mathrm{n}}>5 \mathrm{~K}$ and $35 \mathrm{~K}<T_{\mathrm{e}}<4000 \mathrm{~K}$. More "accurate" cooling rates can be obtained from Fig. 4 ; however, the basic limitation of accuracy is the accuracy of the cross section formula taken from Itikawa (1972). The error in this theoretical formula is not known but it is certainly not better than a few percent.

Rotational cooling rates for several neutral temperatures (but none less than $200 \mathrm{~K}$ ) were presented in Fig. 1 of Ashihara (1975). Both Ashihara's (1975) and our calculations employed the same (Itikawa, 1972 ) cross section formula. Figure 5 is a comparison of our cooling rate from equation (8) and Ashihara's (1975) for $T_{\mathrm{n}}=300 \mathrm{~K}$ and there are significant differences; however, if one shifts the curve by $300 \mathrm{~K}$ the agreement is better than $15 \%$, which no doubt implies that the correct abscissa in Fig. 1 of Ashihara is really $T_{\mathrm{e}}-T_{\mathrm{n}}$ rather than $T_{\mathrm{c}}$.

Ashihara (1975) presented an analytical formula for electron rotational cooling by symmetric-top molecules and suggested that this could also be applied

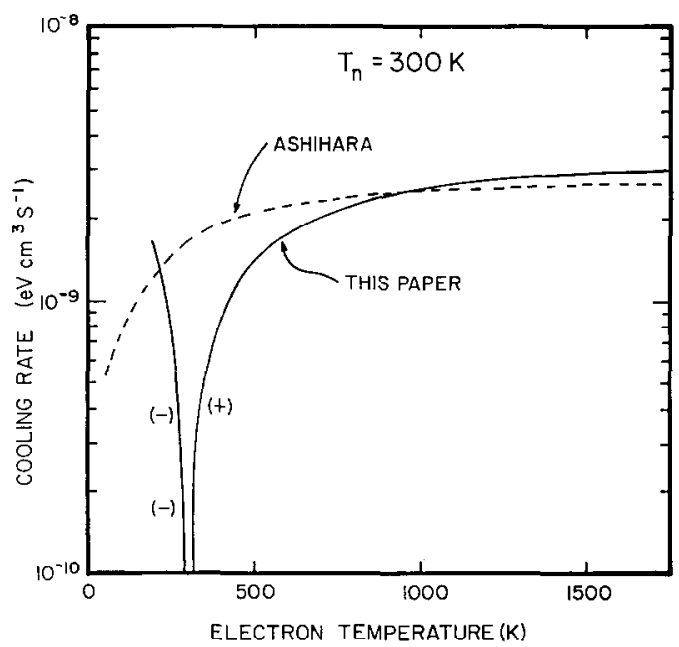

Fig. 5. COMParison of ROTATIONAL COOLING RATES From THIS PAPER AND FROM FIG. 1 OF ASHIHARA (1975) FOR $T_{\mathrm{n}}=$ $300 \mathrm{~K}$

The curves are labeled with $(+)$ or $(-)$ depending on whether or not the cooling rate is positive or negative. to asymmetric top molecules with an appropriate assignment of rotational constants. Following this procedure Marconi and Mendis (1984) and Mendis et al. (1985) generated $\mathrm{H}_{2} \mathrm{O}$ cooling rates which agree with ours to within about $20 \%$ for $T_{\mathrm{e}}>T_{\mathrm{n}}$ and $T_{\mathrm{n}}>100 \mathrm{~K}$ and $T_{\mathrm{e}}$ less than a few thousand degrees. Our cooling rates are almost a factor of two larger than those given by the Ashihara expression for $T_{\mathrm{e}}=10^{4} \mathrm{~K}$.

\section{VIBRATIONAL EXCITATION OF WATER VAPOR BY ELECTRON IMPACT}

Several types of vibrational excitation are possible for the $\mathrm{H}_{2} \mathrm{O}$ molecule the bending mode and the symmetric and asymmetric stretching modes. Seng and Linder (1976) measured cross sections for the electron impact excitation of the $(000)$ to $(010)$ transition and the combined (000) to (100) and (001) transitions. The thresholds for these three transitions are $0.198,0.453$ and $0.466 \mathrm{eV}$, respectively. These vibrational excitation cross sections exhibit resonance behavior and are very large (i.e. about $3 \times 10^{-16} \mathrm{~cm}^{2}$ ), just above the threshold energies. The Seng and Linder cross sections are not well resolved near the thresholds; however, Rohr (1977) has published detailed cross section data near the threshold for the combined (000)-(001) and (100) transitions, albeit only for one scattering angle $\left(\theta=120^{\circ}\right)$.

Vibrational excitation cross sections for lower energies are shown in Fig. 6. At higher energies the cross sections are from Seng and Linder (1975), but we used the relative variation of the Rohr (1977) data for the $(000)-(001,100)$ transition at lower energies near the threshold, as well as for the (000)-(010) transition. We need an analytic form for electron energies greater than $10 \mathrm{eV}$. For these energies the form introduced by Green and Dutta (1967) asymptotically becomes a simple power function :

$$
\begin{aligned}
\sigma_{001}+\sigma_{100} & =1.96 \times 10^{-16} / E^{0.75} \\
\sigma_{010} & =8.43 \times 10^{-17} / E^{0.75} .
\end{aligned}
$$

Equation (9) is designed to give reasonable cross sections only for energies greater than $10 \mathrm{eV}$ and which match the measured cross sections for energies near $10 \mathrm{eV}$. For energies below $10 \mathrm{eV}$, the measured cross sections (Fig. 6 for low energies and Seng and Linder, 1976, for intermediate energies) should be used.

\section{VIBRATIONAL COOLING OF ELECTRONS BY WATER VAPOR}

The vibrational cooling rate, $R_{v}\left(T_{\mathrm{c}}, T_{\mathrm{n}}\right)$, can be calculated using equation (7) but with a vibrational 

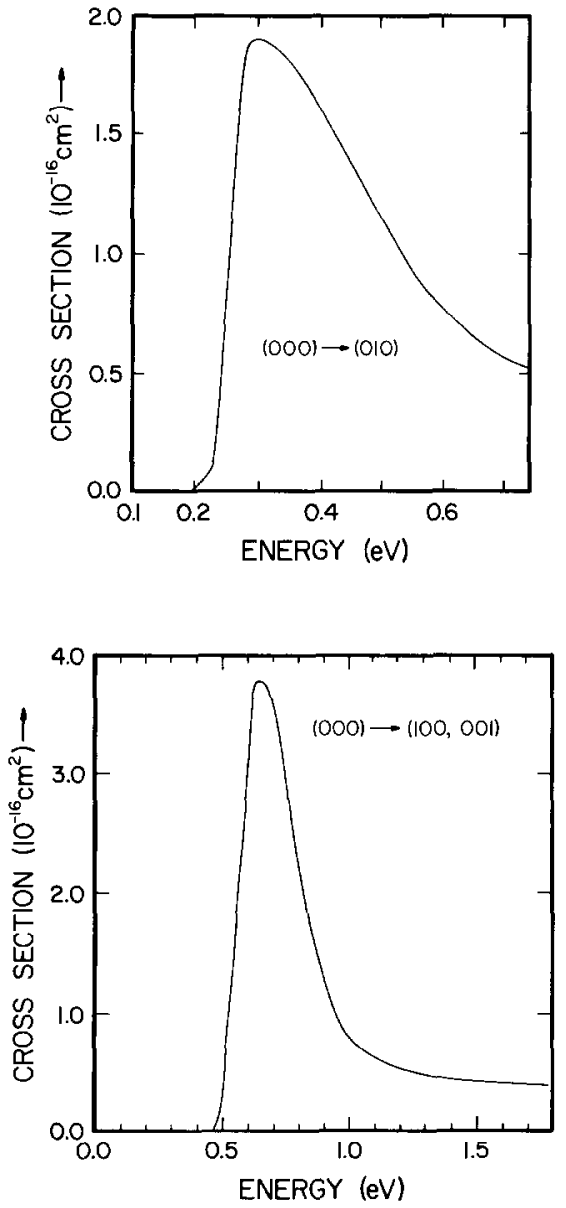

Fig. 6. Cross SECtions versus ENERGy For the vibrational EXCITATION OF $\mathrm{H}_{2} \mathrm{O}$ BY ELECTRONS.

In the top panel, the total cross section for the $(000)-(010)$ transition is shown. In the bottom panel, the total cross section for the combined transitions $(000)-(100,001)$ is shown. These cross sections were obtained by combining the measurements of Seng and Linder (1976) and Rohr (1977).

loss function instead of the rotational loss function. The vibrational loss function, $L_{v j}\left(E, T_{n}\right)$, can be simply calculated using the measured vibrational cross sections in the manner described in Waite and Cravens (1981) for molecular hydrogen. The de-excitation cross section for transition $j$ (i.e. $\sigma_{d i}(E)$ ) can be obtained from the excitation cross section, $\sigma_{i}(E)$, by equating the collision strengths (Mott and Massey, 1965):

$$
\sigma_{d j}(E)=\left(\left(E+W_{j}\right) / E\right) \sigma_{j}(E) .
$$

The partial loss function for vibrational excitation and de-excitation for the transition $j$ can be written
(Waite and Cravens, 1981):

$$
\begin{aligned}
L_{\mathrm{v} j}\left(E, T_{\mathrm{n}}\right)=W_{j}\left\{\sigma_{j}(E)-\exp \left[-W_{j} / k T_{\mathrm{n}}\right]\right. \\
\left.\times\left(\left(E+W_{j}\right) / E\right) \sigma_{j}\left(E+W_{j}\right)\right\}
\end{aligned}
$$

where $W_{j}$ is the threshold for transition $j$ (either 0.198 or $0.46 \mathrm{eV}$ here for $j=1$ or $j=2$ ), and $T_{\mathrm{n}}$ is the neutral temperature (actually the vibrational temperature but we will assume that this is equal to the neutral temperature).

The partial cooling rate for transition $j, R_{v j}\left(T_{\mathrm{e}}, T_{\mathrm{n}}\right)$, is found by combining equations (7) and (11):

$$
\begin{aligned}
R_{v j}\left(T_{\mathrm{e}}, T_{\mathrm{n}}\right) & =\left[8.37 \times 10^{13} W_{j} / T_{\mathrm{e}}^{3 / 2}\right] \\
& \times\left\{1-\exp \left[\left(W_{j} / k\right)\left(T_{\mathrm{e}}^{-1}-T_{\mathrm{n}}^{-1}\right)\right]\right\} I_{j}\left(T_{\mathrm{e}}\right)
\end{aligned}
$$

where the integrals $I_{j}$ are defined as :

$$
I_{j}\left(T_{\mathrm{e}}\right)=\int_{W,}^{\infty} E \sigma_{j}(E) \exp \left[-E / k T_{\mathrm{e}}\right] \mathrm{d} E
$$

The total vibrational cooling rate for $\mathrm{H}_{2} \mathrm{O}$ is :

$$
R_{v}\left(T_{\mathrm{e}}, T_{\mathrm{n}}\right)=R_{v 1}\left(T_{\mathrm{e}}, T_{\mathrm{n}}\right)+R_{v 2}\left(T_{\mathrm{e}}, T_{\mathrm{n}}\right) \quad \mathrm{eV} \mathrm{cm}^{3} \mathrm{~s}^{-1}
$$

where $I_{1}\left(T_{\mathrm{e}}\right)$ and $I_{2}\left(T_{\mathrm{e}}\right)$ were cvaluated numerically using the cross sections from Fig. 6 . These functions of $T_{\mathrm{e}}$ are displayed in Fig. 7. These functions plus

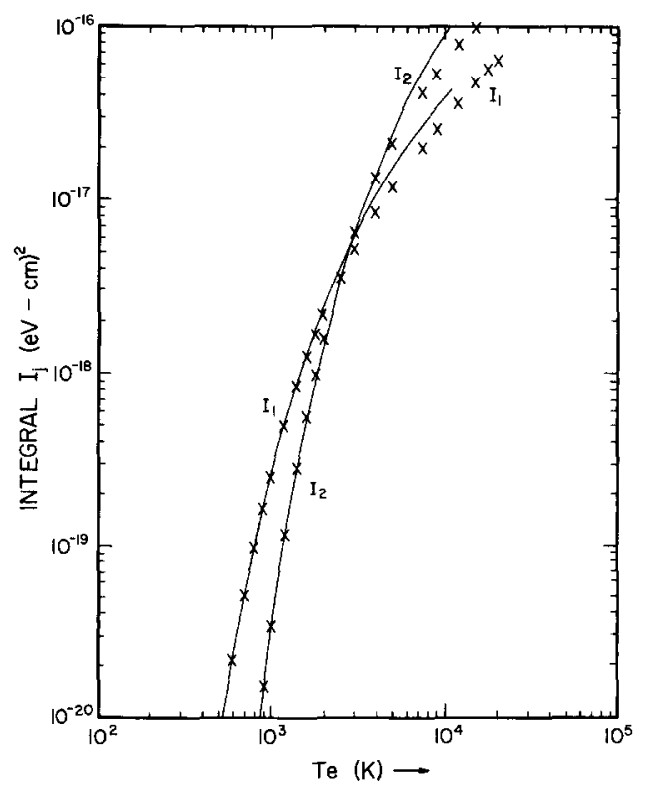

Fig. 7. THE I $_{1}$ AND $I_{2}$ INIEGRALS AS FUNCTONS OH ELECTRON TEMPERATURE ARE SHOWN.

The results of numerical calculations are indicated by the Xs and the analytical fits are given by the solid lines. These functions are used to calculate the vibrational cooling rate of electrons in $\mathrm{H}_{2} \mathrm{O}$. 
equations (12) and (14) give the total vibrational cooling rate. $\left.W_{1}=0.198\right) \mathrm{eV}$ and $W_{2}=0.46 \mathrm{eV}$. $k=8.617 \times 10^{-5} \mathrm{eV} \mathrm{K}^{-1}$ is Boltzmann's constant.

$I_{1}\left(T_{\mathrm{e}}\right)$ and $I_{2}\left(T_{\mathrm{e}}\right)$ were fit to the following analytic formulae in order to make the cooling rate easier to use in a practical application:

$$
I_{j}\left(T_{\mathrm{e}}\right)=\exp \left\{A_{j}+B_{j} / T_{\mathrm{e}}^{1 / 2}+C_{j} / T_{\mathrm{e}}^{3 / 2}+D_{j} / T_{\mathrm{e}}^{2}\right\}
$$

with

$$
\begin{array}{cl}
A_{1}=-35.62 & B_{1}=-215.0 \\
A_{2}=-33.91 & B_{2}=-297.0 \\
C_{1}=-1.75 \times 10^{4} & D_{1}=5.25 \times 10^{4} \\
C_{2}=-6.11 \times 10^{4} & D_{2}=2.66 \times 10^{5}
\end{array}
$$

These analytic fits are good to about $10 \%$ or better (Fig. 7) for $T_{\mathrm{c}}$ in the range $300-3000 \mathrm{~K}$. The agreement is somewhat worse for higher or lower values of $T_{\mathrm{e}}$ and $I_{1}\left(T_{\mathrm{e}}\right)$ and $I_{2}\left(T_{\mathrm{e}}\right)$ should then be taken directly from Fig. 7 if one requires a high degree of accuracy.

The vibrational cooling rate from equation (12) with values of $I_{1}\left(T_{\mathrm{e}}\right)$ and $I_{2}\left(T_{\mathrm{e}}\right)$ taken from equation (15), are shown in Fig. 8 as a function of $T_{\mathrm{e}}-T_{\mathrm{n}}$ for $T_{\mathrm{n}}=100$ and $300 \mathrm{~K}$. The vibrational cooling rate increases sharply as $T_{\mathrm{c}}-T_{\mathrm{n}}$ increases. Comparing the rotational and vibrational cooling rates (Figs 4 and 8 , respectively), one sees that rotational cooling is more

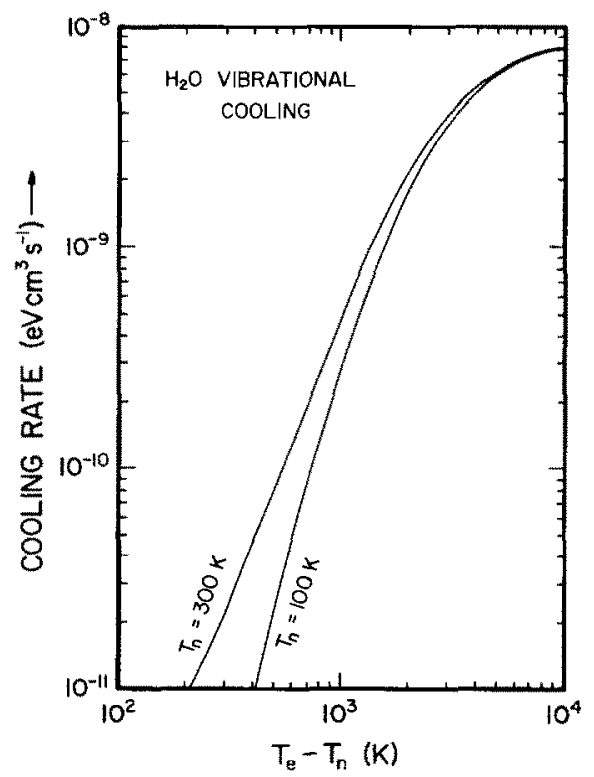

FIG. 8. CALCULATED VIBRATIONAL COOLING RATES IN WATER VAPOR VERSUS THE DIFFERENCE BETWEEN THE ELECTRON AND THE NFUTTRAL TEMPFR ATURE FOR THE VALUES OF THE NEUTRAL TEMPERATURE SHOWN.

The analytical fits were used for the $I_{1}$ and $I_{2}$ functions. important for $T_{\mathrm{e}}$ less than about $2500 \mathrm{~K}$ and vibrational cooling is more important for higher temperatures. The total electron cooling rate is ahout 100 times larger for $\mathrm{H}_{2} \mathrm{O}$ than it is for a non-polar molecule like $\mathrm{H}_{2}$ (Waite and Cravens, 1981).

The vibrational cooling rates given by the expression in Ashihara (1975) are much smaller than those calculated in this paper for $T_{\mathrm{e}}-T_{\mathrm{n}}>100 \mathrm{~K}$. The reason is that the Born approximation cross sections implicit in the Ashihara expression do not include the contributions of resonances which are very important and which are apparent in the measured cross sections (Seng and Linder, 1976).

A further comment should be made concerning rotational and vibrational cooling of electrons by $\mathrm{H}_{2} \mathrm{O}$. The electron heat lost to $\mathrm{H}_{2} \mathrm{O}$ appears as rotational or vibrational excitation, and this energy is either converted to translational energy via neutralneutral collisions or is lost to the system via the emission of long wavelength radiation. In an active comet, at least the inner coma is usually optically thick to this radiation at the wavelengths of the relevant transitions; this will have the effect of increasing the neutral temperature over what it would be for an optically thin coma (Crovisier, 1984 ; private communication, Marconi, M., 1985). Nonetheless, as far as the electrons are concerned, the rotational and vibrational cooling rates can be specified in terms of the electron and neutral (rotational/vibrational) temperatures, without it being necessary to know the ultimate fate of the heat lost by the electrons.

\section{ION-NEUTRAL COLLISIONS AND COOLING RATE}

Ion-neutral interactions are an important aspect of both the dynamics and the energetics of ionospheres (cf. Banks and Kockarts, 1973) including cometary ionospheres (Mendis et al., 1985; Cravens et al., 1983; Korosmezey, 1985). For instance, ion-neutral interactions strongly couple the ion and neutral motions in the inner coma. The ion-neutral momentum transfer collision frequency is required for ionospheric calculations. The ion-neutral energy transfer rate (or cooling rate), $R_{\text {in }}$, can be specified in terms of the momentum transfer collision rate constant, $k_{\text {in }}$, by using equation (22.84) from Banks and Kockarts (1973):

$$
\begin{aligned}
& R_{\text {in }}=-2.6 \times 10^{-4} k_{\text {in }} \mu_{\mathrm{A}} \\
& \times\left(T_{\mathrm{i}}-T_{\mathrm{n}}\right) /\left(A_{\mathrm{i}}+A_{\mathrm{n}}\right) \quad \mathrm{eV} \mathrm{cm}^{3} \mathrm{~s}^{-1}
\end{aligned}
$$

where $\mu_{\mathrm{A}}$ is the ion-neutral reduced mass, and $A_{i}$ and $A_{\mathfrak{n}}$ are the ion and neutral masses (in a.m.u. units), respectively. The ion-neutral interaction of most 
importance for collisions of point ions with non-polar molecules is the induced dipole interaction which has the following potential, $V(r)$, as a function of the internuclear separation, $r$ :

$$
V(r)=-\alpha e^{2} / 2 r^{4}
$$

where $\alpha$ is the polarizability of the neutral molecule and $e$ is the electric charge (Banks and Kockarts, 1973; Dugan and Magee, 1967; Hyatt and Stanton, 1970). From this potential one can derive a momentum transfer cross section which is proportional to $(\alpha / E)^{1 / 2}$ where $E$ is the energy of the relative motion of the ion and the neutral target. The momentum transfer rate constant (essentially the "Langevin" rate constant $k_{\mathrm{L}}$ ) can be derived from this cross section (Banks and Kockarts, 1973):

$$
k_{\mathrm{L}}=2.6 \times 10^{-9}\left(\alpha / \mu_{\mathrm{A}}\right)^{1 / 2} \mathrm{~cm}^{3} \mathrm{~s}^{-1}
$$

where $\mu_{\mathrm{A}}$ is the ion-neutral reduced mass in a.m.u.

The ion-neutral collision frequency for a polar molecule like $\mathrm{H}_{2} \mathrm{O}$ is expected to be much larger than the Langevin collision frequency (the collision frequency is $k_{1} n_{\mathrm{n}}$ where $n_{\mathrm{n}}$ is the neutral density). The interaction potential for an ion interacting with a permanent dipole can differ markedly from the induced dipole potential given by equation (17). The maximum effect is achieved if the target dipole is "locked" and follows the colliding ion along its trajectory, which is likely only if the relative velocity is rather small and if the target rotational motion is small. In this case, the potential varies as $1 / r^{2}$ and the cross section varies as $1 / E$ (Dugan and Magee, 1967). However, for large relative velocities and/or large rotational energies, the effective interaction potential is again the induced dipole potential. And at very large relative velocities the charge transfer process dominates for momentum transfer in any case (Banks and Kockarts, 1973).

Chesnavich et al. (1978) have reviewed various theories for ion-dipole collisions in the context of "capture" rate constants and they provide a convenient approach for this type of interaction. The momentum transfer collision rate constant should be approximately the same as the capture rate constant. The most convenient formulae given in Chesnavich $e t$ $a l$. are formulated in terms of the temperature $T$, and assume that the ion and neutral kinetic temperatures and the rotational temperature are all the same. However, we will adopt these formulae even for unequal ion and neutral temperatures by defining $T$ as:

$$
T \approx 0.3 T_{\mathrm{i}}+0.7 T_{\mathrm{n}}
$$

where each component was weighted with the relevant degrees of freedom. The rotational temperature was assumed to be the same as the neutral kinetic temperature $T_{\mathrm{n}}$.

Adiabatic theory (see Chesnavich et al., 1978, and references therein) provides the following capture rate constant relative to $k_{\mathrm{L}}$ :

$$
\begin{aligned}
k_{\mathrm{ADI}} / k_{\mathrm{L}}=1.0+\left\{1.0 / T_{\mathrm{R}}^{1 / 2}+\right. & T_{\mathrm{R}}^{1 / 2}\left(1-\exp \left(T_{\mathrm{R}}^{-1}\right)\right) \\
& \left.-\pi^{1 / 2} \operatorname{erf}\left(1.0 / T_{\mathrm{R}}^{\mathrm{I} / 2}\right)\right\}
\end{aligned}
$$

where the reduced temperature is defined by:

$$
T_{\mathrm{R}}=2 \alpha k T / D^{2} \quad\left(=T(\mathrm{~K}) / 8588 \mathrm{~K} \text { for } \mathrm{H}_{2} \mathrm{O}\right) \text {. }
$$

Only the first term in the brackets of equation (20) is important for small values of the reduced temperature. Equation (20) gives $k_{\mathrm{ADI}} / k_{\mathrm{L}}=2.5$ for water and for $T=300 \mathrm{~K}$.

Variational theory (again see Chesnavich et al., 1978) provides an upper limit to the "true" capture rate constant. The following formula is appropriate for small values of $T_{\mathrm{R}}$ (Chesnavich et al., 1978):

$$
k_{\mathrm{VAR}} / k_{\mathrm{L}} \approx 9\left(1+2 T_{\mathrm{R}}\right) /\left[8\left(\pi T_{\mathrm{R}}\right)^{1 / 2}\right]
$$

$\mathrm{k}_{\mathrm{VAR}} / k_{\mathrm{L}} \approx 3.6$ for water and for $T=300 \mathrm{~K}$. A different formula applies for large values of the reduced temperature.

The experimental values of the capture rate which are shown in Fig. 3 of Chesnavich et al. mostly lie above the adiabatic rate, $k_{\mathrm{AD}}$, but below the variational rate, $k_{\mathrm{VAR}}$. Huntress ef al. (1980) have measured the reaction rate for the following reaction at $300 \mathrm{~K}$ :

$$
\begin{aligned}
& \mathrm{H}_{2} \mathrm{O}^{+}+\mathrm{H}_{2} \mathrm{O} \rightarrow \mathrm{H}_{3} \mathrm{O}^{+}+\mathrm{OH} \\
& k_{23}=2.05 \times 10^{-9} \mathrm{~cm}^{3} \mathrm{~s}^{-1} .
\end{aligned}
$$

The Langevin rate constant from equation (18) is $1.05 \times 10^{-9} \mathrm{~cm}^{3} \mathrm{~s}^{-1}$, giving a ratio of $k_{23} / k_{\mathrm{L}}-2$. Of course, $k_{23}$ does not include any non-reactive collisions and is a lower limit to the total collision frequency. Nonetheless, both this reaction and the Chesnavich et al. paper indicate that the polar nature of the water molecule enhances the collision frequency by approximately a factor of 2 at $T \approx 300 \mathrm{~K}$, and equations (20) and (22) indicate that the enhancement can be considerably larger than a factor of 2 for smaller values of $T$.

The polar nature of the water molecule can be approximately taken into account in models of conetary ionospheres by using the original Banks and Kockarts (1973) ion-neutral momentum transfer collision frequency (also given by equation (17)) multiplied by a correction factor, $C$, which can approximately be defined as the average of $k_{\mathrm{ADI}}$ and $k_{\mathrm{VAR}}$ :

$$
C \approx 0.5\left(k_{\mathrm{ADI}} / k_{\mathrm{L}}+k_{\mathrm{VAR}} / k_{\mathrm{L}}\right) \text {. }
$$


Equations (20) and (22) can be used in conjunction with equation (24) to obtain values for $C$ which are appropriate to lower values of $T$; formulae in Chesnavich $e t$ al. can be used in $k_{\mathrm{VAR}}$ for larger values of $T$. However, for larger $T$, one expects $C$ to approach unity and charge exchange will begin to dominate for momentum transfer in any case.

\section{SUMMARY}

Cooling rates for electrons and ions in water vapor are required in order to understand the energetics of cometary ionospheres. In particular, these cooling rates are needed to calculate electron and ion temperatures. These electron and ion temperatures can be used to calculate the total ionospheric plasma pressure, which in turn can be used to determine the location of the cometary ionopause. In this paper, both rotational and vibrational cooling rates for electrons were calculated. Convenient analytical formulae for these cooling rates were provided for a wide range of electron and neutral temperatures. Some relatively recent work in the literature on ion-neutral collisions for polar molecules was reviewed, and a momentum transfer collision frequency was obtained in a form which is useful (albeit approximate) for ionospheric calculations. This momentum transfer collision frequency can be used to obtain the ion-neutral energy transfer rate (or cooling rate).

Acknowledgements - The authors thank R. Nightingale for computational assistance. This work was supported by NASA Grants NAGW-15 and NGR 23-005-015 and NSF Grant ATM 8417884.

\section{REFERENCES}

Ashihara, O. (1975) The electron energy loss rates by polar molecules. Inst. Space and Aeronautical Sci. University of Tokyo Report No, $53040,10$.

Ashihara, $0 .(1976)$ Photoelectron fuxes in cometary atmospheres. Icarus 35, 369.

Banks, P. M. and Kockarts, G. (1973) Aeronomy. Academic Press, New York

Chesnavich, W. J., Su, T. and Bowers, M. T. (1978) Iondipole collisions: Recent theoretical advances, in Kinetics of Ion-Molecule Reactions (Edited by Ausloos, P.). NATO Advanced Study Institutes Series, LaBaule.

Cravens, T. E., Gombosi, T. I., Horanyi, M., Kecskemety, K., Korosmezey, A., Marconi, M. L., Mendis, D. A., Nagy, A. F., Sagdeev, R. Z., Shevchenko, V. I., Shapiro, V. D. and Szego, K. (1983) The role of electric fields in the cometary environment, KFKI preprint. Report of the International Theoretical Workshop on Comets, Budapest.

Cross, P. C., Hainer, R. M. and King, G. W. (1944) The asymmetric rotor. II. Calculations of dipole intensities and line classification. $J$. chem. phys. 12, 210.
Crovisier, J. (1984) The water molecule in comets: Fluorescence mechanisms and thermodynamics of the inner coma. Astron. Astrophys. 130,361.

Dalby, F. W. and Nielson, H. H. (1956) Infrared spectrum of water vapor. Part 1. The $6.26 \mu$ region. J. chem. Phys. 25, 934.

Delsemme, A. H. (1982) Chemical composition of cometary nuclei, in Comets (Edited by Wilkening, L. L.). The University of Arizona Press, Tucson, Arizona.

Dugan, J. V., Jr. and Magee. J. L. (1967) Capture collisions between ions and polar molecules. J. chem. Phys. 47, 3103.

Galeev, A. A., Cravens, T. E. and Gombosi, T. I. (1985) Solar wind stagnation near comets. Astrophys, J. 289, 807.

Green, A. E. S. and Dutta, S. K. (1967) Semi-empirical cross sections for electron impact. $J$. geophys. Res. 72, 3933.

Henry, R. J. W. and MeElroy, M. B. (1969) The absorption of extreme ultraviolet radiation by Jupiter's upper atmosphere. $J$. atmos. Sci. 26, 912.

Huntress, W., McEwan, M. J., Karpas, Z. and Anicich, V. G. (1980) Laboratory studies of some of the major ion-molecule reactions occurring in the cometary comae. Astrophys. J. Suppl. Ser. 44, 481.

Hyatt, D. and Stanton, L. (1970) Application of a multipole potential in a theoretical investigation of collision crosssections for ions with linear molecules. Proc. R. Soc. 318 , 107.

Ip, W.-H. and Axford, W. I. (1982) Theories of physical processes in the cometary comae and in tails, in Comets (Edited by Wilkening, L. L.). The University of Arizona Press, Tucson, Arizona.

Itikawa, Y.J. (1972) Rotational transition in an asymmetrictop molecule by electron collision: Applications to $\mathrm{H}_{2} \mathrm{O}$. J. phys. Soc. Japan 32, 217.

Jain, A. and Thompson, D. G. (1983) Rotational excitation of $\mathrm{CH}_{4}$ and $\mathrm{H}_{2} \mathrm{O}$ by slow electron impact. J. Phys. $B: A t$. Mol. Phys. 16, 3077.

Jung, K., Antoni, T., Muller, R., Kochem, K.-H. and Ehrhardt, H. (1982) Rotational excitation of $\mathrm{N}_{2}, \mathrm{CO}$ and $\mathrm{H}_{2} \mathrm{O}$ by low-energy electron collisions. I. Phys. $B: A t$. Mol. Phys. 15, 3535 .

King, G. W., Hainer, R. M. and Cross, P. C. (1947) Expected microwave absorption coefficients of water and related molecules. Phys. Rev. 71, 433.

Korosmezey, A. (1985) Modeling of cometary surfaces and inner coma. Ph.D. thesis, Roland Eotvos University.

Marconi, M. L. and Mendis, D. A. (1984) The effects of the difuse radiation fields due to multiple scattering and thermal re-radiation by dust on the dynamies and thermodynamics of a dusty cometary atmosphere. Astrophys. J. 287, 445.

Mendis, D. A., Houpis, H. L. F. and Marconi, M. L. (1985) The physics of comets. Fundamentals of Cosmic Phys. 10, 1 .

Mott, N. F. and Massey, H. S. W. (1965) The Theory of Atomic Collisions. Clarendon Press, Oxford.

Rohr, K. (1977) Characteristics of the threshold structures in the $\mathrm{e}-\mathrm{H}_{2} \mathrm{O}$ vibrational excitations. J. Phys. B: At. Mol. Phys. 10, L735.

Schmidt, H. U. and Wegmann, R. (1982) Plasma flows and magnetic fields, in Comets (Edited by Wilkening, L. L.). The University of Arizona Press, Tucson, Arizona.

Seng, G. and Linder, F. (1976) Vibrational excitation of polar molecules by electron impact 11 . Direct and resonant excitation in $\mathrm{H}_{2}$ O. J. Phys. B : At. Mol. Phys. 9, 2539. 
Takayanagi, K. and Itikawa, Y. (1970) The rotational excitation of molecules by slow electrons. Adv. atomic molec. Phys. 6, 105.

Van Vleck, J. H. (1947) The absorption of microwaves by uncondensed water vapor. Phys. Rev. 71, 425.

Waite, J. H., Jr. and Cravens, T. E. (1981) Vibrational and rotational cooling of electrons by molecular hydrogen. Planet. Space Sci. 29, 1333.

Wallis, M. K. and Ong, R. S. B. (1976) Cooling and recombination processes in cometary plasma, in The Study of Comets. NASA SP-393 (Edited by Donn, B., Mummo, M., Jackson, W., A'Hearn, M. and Harrington, R.) p. 856 . 